\title{
Trastuzumab-Doxorubicin Conjugate Provides Enhanced Anti-Cancer Potency and Reduced Cardiotoxicity*
}

\author{
Ningyan Zhang ${ }^{1 \#}$, Melvin E. Klegerman ${ }^{2}$, Hui Deng ${ }^{1}$, Yun Shi ${ }^{1}$, Eva Golunski ${ }^{2}$, Zhiqiang An ${ }^{1 \#}$ \\ ${ }^{1}$ Texas Therapeutics Institute, Brown Foundation Institute of Molecular Medicine, The University of Texas Health Science Center at \\ Houston, Houston, USA; ${ }^{2}$ Division of Cardiovascular Medicine, Department of Internal Medicine, University of Texas Health Sci- \\ ence Center, Houston, USA. \\ Email: "Ningyan.zhang@uth.tmc.edu
}

Received December $27^{\text {th }}, 2012$; revised January $25^{\text {th }}, 2013$; accepted February $5^{\text {th }}, 2013$

\begin{abstract}
Since trastuzumab monotherapy for treatment of breast cancer with HER2/ErbB2 over-expression has been shown to have limited efficacy, combined treatment of trastuzumab with chemotherapy is widely practiced in clinic. However, certain combination treatments of trastuzumab and chemotherapy (i.e. doxorubicin) are not recommended due to high risk of cardiotoxicity. Antibody-drug conjugates (ADCs) offer selective delivery of cytotoxic agents into targeted cancer cells, thereby allowing for reduced general cellular cytotoxicity caused by chemotherapeutic agents through antibody mediated specific recognition of tumor antigens. In this study, we constructed a trastuzumab-doxorubicin conjugate (T-Dox) using a thioether linkage and characterized both biophysical stability and anti-cancer potency of the T-Dox using a panel of HER2 expressing cancer cell lines. The T-Dox conjugate showed significantly improved anti-cancer potency in comparison with trastuzumab. The results demonstrated for the first time that there were significant differences in the uptake of T-Dox among high HER2 expression cancer cells and higher T-Dox uptake also showed stronger anti-cancer potency. Similar to trastuzumab, T-Dox selectively bound to HER2 overexpressing cancer cells and low HER2 expression cells had no detectable uptake of T-Dox. Consistent to the uptake data, human cardiomyocyte cells had no detectable HER2 expression and T-Dox showed minimal cytotoxic effects. On the contrary, a treatment with combination of trastuzumab and doxorubicin showed severe cytotoxicity to human cardiomyocytes $(>90 \%$ cell death after 3 day exposure). This study demonstrated that trastuzumab conjugated with doxorubicin (T-Dox) can provide valuable alternative to the combination treatment with doxorubicin and trastuzumab for high HER2 expressing cancer patients.
\end{abstract}

Keywords: Antibody Drug Conjugate; ADC; Trastuzumab; Doxorubicin; Cardiotoxicity; HER2/ErbB2 Internalization

\section{Introduction}

Doxorubicin is a DNA intercalating agent that has been used as an effective chemotherapeutic for treatment of many types of cancer, including breast cancers. However, its use is severely limited due to cardiotoxicity, especially the risk of developing congestive heart failure with high cumulative doses $\left(>400 \mathrm{mg} / \mathrm{m}^{2}\right)$ [1]. Extensive efforts have been made to reduce cardiotoxicity and to improve the therapeutic index of doxorubicin [2-9]. Various delivery methods including liposomal polymers were investigated to improve the pharmacokinetics of doxorubicin in vivo [10-15]. Targeted delivery of doxorubicin using nanoparticles is being tested in clinical trials [16].

Antibody drug conjugation is becoming a prominent

*Funding source: This work was supported in part by a grant from the Texas Emerging Technology Fund; the Welch Foundation Grant AU00024; and National Institute of Health, R01 HL74002.

${ }^{\#}$ Corresponding authors. approach for targeted delivery of cytotoxic agents including doxorubicin [17-19]. The trastuzumab-emtansine conjugate (T-DM1) which is currently in clinical trials has shown a markedly improved clinical efficacy when compared to trastuzumab alone in treatment of metastatic breast cancer, including the inhibition of lapatinib-insensitive metastatic breast cancer [20].

Early studies using combinations of trastuzumab with doxorubicin (anthracycline) demonstrated better clinical efficacy in both total response rate (56\% vs. $17 \%$ - $42 \%$ ) and survival (26.8 months vs. 18.4 - 22.1) when compared with trastuzumab combinations with other agents or single agent chemotherapies, but the combined treatment of trastuzumab and doxorubicin was associated with a high incidence of cardiotoxicity [21]. Antibody drug conjugates (ADC) combine the advantages of antigenantibody specificity and potent cytotoxic effects of chemotherapeutic agents. The positive results from the 
trastuzumab-emtansine (T-DM1) clinical trials indicate that trastuzumab ADC is a viable approach for targeting HER2 expressing cancer cells. In comparison with emtansine which inhibits microtubules, doxorubicin functions as a cytotoxic agent by intercalating DNA. Doxorubicin is an established clinical chemotherapeutic agent, while emtansine is not approved for clinical use as a monotherapy because of its unacceptable safety profile. Pursuing an analogous strategy to the trastuzumab-emtansine conjugate (T-DM1), we chose to couple the carbonyl function of doxorubicin to trastuzumab via a thioether linkage, using the maleimidohydrazide linker MMCCH (SMCC hydrazide) [22].

Among many factors impacting the efficacy and safety of ADCs, antibody mediated selective uptake and linker stability play critical roles in the success of ADC therapies. It was reported that trastuzumab is endocytosed passively through HER2 receptor internalization [23] and it is assumed that trastuzumab-DM1 conjugate is also internalized by the same mechanism [24]. However, factors affecting uptake of trastuzumab drug conjugate through HER2 antigen is not well understood. This study employed a trastuzumab-doxorubicin conjugate (T-Dox) to investigate trastuzumab ADC internalization mechanisms and to evaluate the selectivity and potency of the trastuzumab conjugated doxorubicin in comparison with free doxorubicin, trastuzumab alone and combination of both therapeutic agents.

\section{Material and Methods}

\subsection{Cells and Reagents}

Cancer cell lines MCF7, Skov3 and T47D were from American Type Culture Collection (Manassas, VA, USA) and maintained in recommended culture conditions at $37^{\circ} \mathrm{C}$ in an atmosphere of $5 \% \mathrm{CO}_{2}$. MCF7/HER2 was obtained as described previously [25]. Human immunoglobulin $\mathrm{G}(\mathrm{IgG})$ was from Jackson Immuno Research (West Grove, PA) and trastuzumab antibody obtained from a biological specialty [26]. Doxorubicin was from Sigma-Aldrich (St. Louis, MO). The chemical linkers 4-(4-N-maleimidomethyl) cyclohexane-1-carboxyl hydrazide (MMCCH or SMCC hydrazide) hydrochloride and 3-(2-pyridyldithiolpropionic acid)-N-hydroxysuccinimide ester (SPDP) were from CarboMer, Inc. (San Diego, CA), and Pierce Chem. Co. (Rockford, IL), respectively. Cell culture media RPMI (Roswell Park Memorial Institute) were purchased from Thermo Fisher Scientific (Pittsburgh, PA).

\subsection{Preparation of Doxorubicin-Immunoglobulin Conjugates}

A maleimido derivative of doxorubicin was prepared by a modification of the method of Griffiths et al. [22]. Doxorubicin $(1.23 \mathrm{mg})$ was mixed with 4-(4-N-maleimidomethyl) cyclohexane-1-carboxyl hydrazide hydrochloride $(4.15 \mathrm{mg})$ in a total volume of $0.25 \mathrm{ml} \mathrm{di}$ methylsulfoxide and incubated for 30 minutes at $50^{\circ} \mathrm{C}$. The reaction mixture was loaded onto a preparative $\mathrm{C} 18$ HPLC column $(6 \times 300 \mathrm{~mm}$ YMC ODS-A $5 \mu \mathrm{m}$; Waters Corp., Milford, MA) with 1:1 methanol/water containing $0.1 \%$ trifluoroacetic acid, followed by methanol to elute the free SMCC hydrazide. The doxorubicin-MMCCH was eluted with the loading solvent mixture, dried per vacuo and stored desiccated at $4^{\circ} \mathrm{C}$ until use.

Human $\mathrm{IgG}$ or trastuzumab ( $2 \mathrm{mg}$ ) was thiolated, using SPDP as previously described [27]. The doxorubicin-MMCCH was dissolved in $1-2 \mathrm{ml} 0.05 \mathrm{M}$ acetatebuffered saline with $2 \mathrm{mM}$ EDTA, $\mathrm{pH} 5.3$, and mixed with same volume of the thiolated protein in the same buffer after adding dimethylformamide to a final concentration of $15 \%-20 \%$ and a total volume of $\leq 4.0 \mathrm{ml}$. The mixture was allowed to react for 15 minutes on ice and passed through $2 \mathrm{Zeba}^{\mathrm{TM}}$ spin columns ( $\leq 2 \mathrm{ml} /$ column) at $1200 \times \mathrm{g}$ for 2 minutes, followed by dialysis (MWCO 12 - $14 \mathrm{kDa}$ cellulose) in 100 volumes of $0.02 \mathrm{M}$ phos-

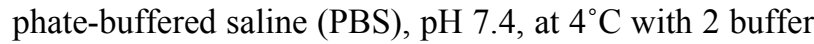
exchanges over a period of 24 hours to remove unconjugated doxorubicin and linker. The dialysis retentate was concentrated to an $\mathrm{IgG}$ concentration of $1 \mathrm{mg} / \mathrm{ml}$ and $\geq$ $30 \mu \mathrm{M}$ doxorubicin, sterilized by passing through a 0.45 $\mu \mathrm{m}$ pore-size syringe filter and lyophilized. The resultant conjugate was stored in a desiccator at $4^{\circ} \mathrm{C}$ until analysis.

\subsection{Determination of Doxorubicin-Antibody Stability at Different Temperatures}

A sterile aliquot $(0.2 \mathrm{ml})$ from a batch of IgG-doxorubicin (IgG-Dox) consisting of $8.8 \mu \mathrm{M}$ doxorubicin conjugated to $308 \mu \mathrm{g}$ human $\operatorname{IgG}$ (4.3:1 dox/IgG molar ratio) was diluted threefold with sterile PBS. One half was incubated for 3 days at $37^{\circ} \mathrm{C}$, while the other half was kept for the same period at $4^{\circ} \mathrm{C}$. Five microliters of each sample was loaded onto a Zenix SEC-300 ( $3 \mu \mathrm{M}, 300 \AA) 4.6$ $\times 250 \mathrm{~mm}$ analytic size exclusion HPLC column (Sepax Technologies, Inc., Newark, DE) and eluted with $0.15 \mathrm{M}$

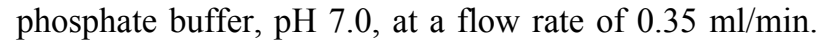
using a Waters 600 controller and Waters 2996 photodiode array detector monitoring absorbance at $214 \mathrm{~nm}$. The column was calibrated with a mixture of thyroglobulin (670 kDa), bovine gamma globulin $(150 \mathrm{kDa})$, ovalbu$\min (45 \mathrm{kDa})$, equine myoglobin $(16.9 \mathrm{kDa})$ and vitamin $\mathrm{B}_{12}(1.35 \mathrm{kDa})$, which produced a linear log-log relationship between molecular weight and elution time from 9.72 to 20.2 minutes (Supplemental Figure 1S). 


\subsection{Determination of Doxorubicin-Antibody Conjugate Stability at Different pH}

To determine stability of the IgG-doxorubicin conjugate at physiological temperature and different $\mathrm{pH}$ conditions, a $1.2 \mathrm{ml}$ sample comprising $11.8 \mu \mathrm{M}$ doxorubicin conjugated to $373 \mu \mathrm{g}$ human $\operatorname{IgG}(4.7: 1 \mathrm{dox} / \mathrm{IgG}$ molar ratio) was divided into two equal aliquots. The $\mathrm{pH}$ of one aliquot was adjusted to 5.4; the other was kept at 7.4. Both aliquots were sterile filtered and lyophilized. Each was divided into 4 equal aliquots after reconstitution and 3 were incubated at $37^{\circ} \mathrm{C}$ for 1,2 and 3 days. One aliquot of each was processed immediately (0-time point). At each time point, two samples were centrifuged at $10,000 \times$ $\mathrm{g}$ for 10 minutes. A portion of each was passed through a micro spin column (Zeba, Pierce Protein Research Products, Rockford, IL) to retrieve the IgG-associated doxorubicin and diluted 3-fold with 0.02 M PBS, pH 7.4. Two aliquots of each sample were assayed for doxorubicin fluorescence (Ex $485 \mathrm{~nm} / \mathrm{Em} 590 \mathrm{~nm}$ ) in a microplate reader (SpectraMax M5, Molecular Devices, Sunnyvale, CA). Replicate measurements were made on successive days to assess inter-assay variance. Duplicate samples were also assayed for protein concentration by optical absorbance at $280 \mathrm{~nm}\left(\mathrm{~A}_{280}\right)$.

\subsection{Determination of HER2 Expression Using a Flow Cytometer}

HER2 expression on different cancer cell lines was determined using a Guava easyCyte HT instrument (Millipore, Danvers, MA) as described before [26]. Trastuzumab was used as primary HER2 staining antibody followed by the addition of phycoerythrin (PE)-conjugated anti-human-Fc (Jackson ImmuneResearch Laboratories, Inc., PA). A human isotype IgG1 antibody was used as reference sample control.

\subsection{Trastuzumab and T-Dox Binding to Cell Surface HER2 Using Fluorescence Imaging}

Binding of trastuzumab and T-Dox to low and high HER2-expressing breast cancer cells was detected using the Olympus IX71 fluorescence microscope (Center Valley, CA). Briefly, cells were seeded in a 6-well-growth chamber and cultured in an incubator at $37^{\circ} \mathrm{C}$ and $5 \%$ $\mathrm{CO}_{2}$ overnight. Trastuzumab or equivalent T-Dox at 1 $\mu \mathrm{g} / \mathrm{ml}$ was added and incubated for 1 hour at $37^{\circ} \mathrm{C}$, followed by the addition of phycoerythrin (PE)-conjugated anti-human-Fc (Jackson ImmunoResearch Laboratories, Inc., West Grove, PA) antibody for detection of trastuzumab. After washing with PBS buffer, cells were visualized under a fluorescence microscope with 20 - 40× objective magnification. Human isotype IgG1 antibody was used as a reference control. Multiple images $(n=3)$ were taken from each well after surveying through each of growth chamber and representative images were documented.

\subsection{Cell Uptake of T-Dox and Doxorubicin Using a Plate Reader}

MCF7/HER2 and Skov3 cancer cells were cultured in RPMI medium with $10 \%$ FBS to $70 \%-80 \%$ confluency and cells were seeded at $100 \mu \mathrm{l}$ media with 10,000 cells/well in a 96 well black plate with clear view bottom (Thermo Fisher Scientific) and cultured overnight at $37^{\circ} \mathrm{C}, 5 \% \mathrm{CO}_{2}$, and $95 \%$ humidity. T-Dox or doxorubicin in media was added into each well at different working concentrations as indicated $(0,0.6,1.8$, and $5 \mu \mathrm{M})$ and cultured for 5 hours in the culture incubator. After the treatment, cells were washed 3 times with cold PBS to remove non-specific carry over T-Dox or doxorubicin on cell surface. Cells were then lysed with RIPA (RadioImmunoprecipitation Assay) buffer (Sigma-Aldrich, St. Luis, MO) containing freshly added protease inhibitor cocktail (Roche Applied Science, Indianapolis, IN) by incubating for 30 minutes on ice and pipetting up and down 5 times during the incubation period. Cell lysates were centrifuged at $3000 \times \mathrm{g}$ for 10 minutes and doxorubicin fluorescence was measured in a plate reader (Molecular Devices, Sunnyvale, CA) at excitation $480 \mathrm{~nm}$ and emission $590 \mathrm{~nm}$.

\subsection{Uptake of T-Dox and Doxorubicin Using Confocal Fluorescence Microscopy}

Cancer cells (MCF7, T47D, MCF7/HER2 and Skov3) with different HER2 expression levels were seeded in each of the designated wells of 8-well chamber slide in RPMI medium with $10 \%$ FBS. After 24 hours incubation at $37^{\circ} \mathrm{C}$ in an atmosphere of $5 \% \mathrm{CO}_{2}$, cells were treated with $5 \mu \mathrm{M}$ of doxorubicin, or doxorubicin equivalent concentrations of T-Dox, and IgG-Dox in the culture medium for 5 hours. Cells were then washed 3 times with PBS and fixed with $4 \%$ formaldehyde in PBS at room temperature (RT) for 20 minutes. Following fixation, cells were washed with PBS and nuclei were stained with 1:1000 diluted TO-PRO-3 (Invitrogen, Carlsbad, CA) in PBS at RT for 10 minutes. After washing, cells were mounted with anti-fade VECTASHIELD mounting medium (Vector Labs, Burlingame, CA). Multiple images were obtained from each well for representation using a $63 \times$ oil immersion objective (NA 1.4) of a Leica TCS SP5 confocal microscope (Leica Microsystems, Germany).

\subsection{Inhibition of Cancer Cell Proliferation}

Cell proliferation and cell survival were assayed as de- 
scribed previously [28] using the xCelligence instrument (Roche Applied Science, Indianapolis, IN). Briefly, cancer cells (5000/well) were seeded in an E-plate (ACEA Biosciences, Inc., CA) and incubated for 4 hours in a cell culture incubator to establish cell baseline growth. Doxorubicin $(0-10 \mu \mathrm{M})$ or doxorubicin equivalent concentrations of T-Dox, IgG-Dox or antibody equivalent concentrations of trastuzumab were added in the E-plate, and cell growth was live monitored through a computer connected with the instrument for 2 - 3 days. Cancer cells only wells $(n=4)$ were used as a baseline control and 1\% Triton X-100 treatment wells were used as the $100 \%$ cell proliferation inhibition control. The Triton-treated cell group has a cell index of zero and the cell index at the beginning of treatment is normalized to a value of 1 . Percentage of cell growth inhibition at the selected time points was calculated using the formula: [(cell index of baseline control group - cell index of treatment group)/cell index of baseline control group] $\times$ 100.

\subsection{Cytotoxicity of Human Cardiomyocyte Cells}

Human primary cardiomyocytes were kindly supplied by Dr. Y. J. Geng, University of Texas Health Science Center at Houston, and cultured in Iscove's Modified Dulbecco's Medium (IMDM) from Gibco (Invitrogen) with $15 \%$ FBS. To start the experiment, cardiomyocyte cells at $70 \%-80 \%$ confluence were seeded $(2000 /$ well $)$ in designated wells of a 96 well E-Plate $(\mathrm{n}=4)$ and incubated for 24 hours at $37^{\circ} \mathrm{C}, 5 \% \mathrm{CO}_{2}$ and $95 \%$ humidity, followed by the addition of trastuzumab or an equivalent concentration of T-Dox, IgG-Dox or a combination of trastuzumab and doxorubicin at $5 \mu \mathrm{M}$ concentration. Cell index was recorded every 30 minutes for 3 days as described above in the cancer cell proliferation assay. Cell viability at the given time points was calculated as (cell index of treated cells/cell index of cardiomyocyte cell control) $\times 100$.

\subsection{Statistical Data Analysis}

All experiments were repeated at least three times and pairwise Student's t-test was conducted when appropriate using GraphPad prism software.

\section{Results}

\subsection{Stability of IgG-Doxorubicin Conjugates}

Doxorubicin and immunoglobulin $\mathrm{G}(\mathrm{IgG})$ conjugates were made in sequential steps as described in the Method section. Activation and addition of chemical linker to doxorubicin is the first key step in the preparation of IgG-doxorubicin conjugates. Complete separation of the un-reacted free chemical linker (eluted before 6 minutes) and doxorubicin (Dox) with linker (eluted after 8 minutes) in the reaction mixture by reverse phase HPLC (C-18 column) is shown in Figure 1(a). Based on the recovered free SMCC hydrazide linker, the coupling process had $41.8 \pm 5.6 \%(\mathrm{SD}, \mathrm{n}=5)$ of the linker coupled to doxorubicin. Free doxorubicin was subsequently removed by size exclusion column separation and dialysis after completion of IgG conjugation. The final T-Dox structure with the chemical linker is shown in Figure 1(b). Characteristics of 4 batches of doxorubicin antibody conjugate preparations ( 2 batches for each of T-Dox and IgGDox) are summarized in Supplemental Table S1; the molar ratio of doxorubicin coupled per molecule of antibody was between 4 and 5 for all four batches indicating the consistency of the conjugation process.

Stability of IgG-Dox was evaluated at $37^{\circ} \mathrm{C}$ and $\mathrm{pH}$ 7.4 (physiological $\mathrm{pH}$ ) or $\mathrm{pH} 5.4$ (lysosomal $\mathrm{pH}$ ) for various time periods of incubation. The results showed that doxorubicin was released from the antibody conjugate slowly at the rate of about $25 \%$ per day due to degradation of the IgG-doxorubicin linkage at $\mathrm{pH}$ 7.4. In contrast, the release of doxorubicin from IgG-Dox was much faster at pH 5.4 with a rate of about $80 \%$ in the first 24 hours incubation, and stabilized at about 20\% IgG-Dox level in the following days (Figure 1(c)). The significant loss of IgGDox at $\mathrm{pH} 5.4$ was mainly due to denaturation of antibody as shown in Figure 1(d). The denatured IgG-Dox at $\mathrm{pH}$ 5.4 was removable as a precipitate by centrifugation at $37^{\circ} \mathrm{C}$, but no loss of protein was noted over a 3-day period at $37^{\circ} \mathrm{C}$, pH 7.4 (Figure 1(d)). Size exclusion chromatograph (SEC)-HPLC analysis confirmed that the trastuzumab remained intact over a period of 3 days at $37^{\circ} \mathrm{C}, \mathrm{pH}$ 7.4 (Supplemental Figure S1).

\subsection{Binding of T-Dox on HER2 Expressing Cancer Cells}

A panel of cancer cells with different levels of HER2 expression from low (MCF7), medium (T47D) to high (MCF7/HER2 and Skov3) was selected to study the effects of HER2 expression levels on T-Dox uptake and its anti-cell proliferative activity. HER2 expression levels measured as relative mean fluorescence levels (MFI) were 3061, 2803, 328, and 112 for Skov3, MCF7/HER2, T47D, and MCF7, respectively (Figure 2(a)). To further determine T-Dox specific binding on cell surface HER2, we stained cancer cells with T-Dox or trastuzumab and detected binding with fluorescence imaging. Similar to trastuzumab, T-Dox selectively bound to HER2 over expressing cancer cells as indicated by the strong fluorescent intensity of T-Dox associated with high HER2 expressing MCF7/HER2 and Skov3 cells, but not detectable on low and medium HER2 expressing MCF7 and T47D cells (Figure 2(b)). 


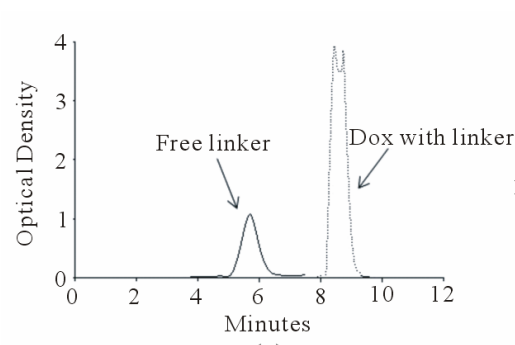

(a)

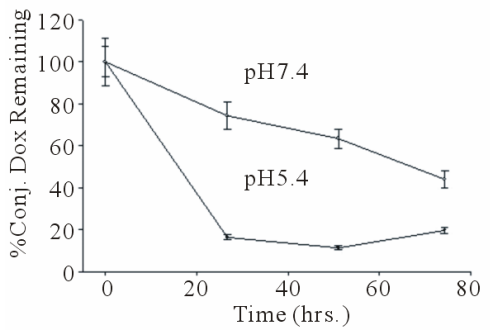

(c)

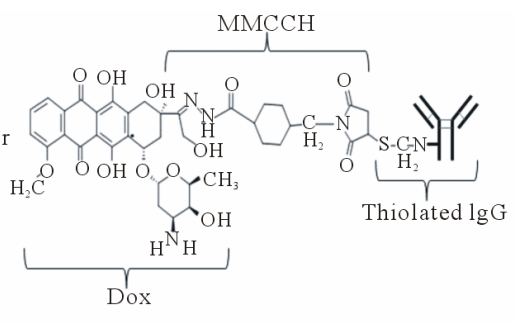

(b)

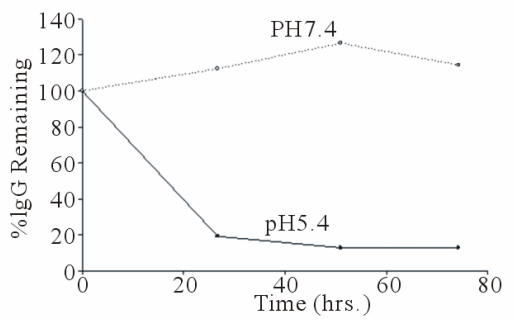

(d)

Figure 1. Construction and characterization of trastuzumab doxorubicin conjugate. (a) HPLC elution profile of MMCCHDoxorubicin reaction mixture applied to preparative $\mathrm{C} 18$ column. The solid line is optical absorbance at $300 \mathrm{~nm}$ wavelength $\left(A_{300}\right)$ for the absorbance maximum of $\mathrm{MMCCH}$; the dotted line is $A_{485}$, near the $A_{\max }$ of doxorubicin. (b) $T$-Dox conjugate linker structure. (c) In vitro stability of human IgG-doxorubicin conjugate (IgG-Dox, batch2). IgG-Dox was determined in the high molecular weight fractions collected in desalting columns after incubation at $37^{\circ} \mathrm{C}$ for varying times under two different pH conditions. Solid circles, pH 5.4; open circles, pH 7.4. (d). IgG-doxorubicin recovery by high speed centrifugation (10,000 $\mathrm{g}$ for $10 \mathrm{~min}$ ) after incubation at $37^{\circ} \mathrm{C}$ for varying times at $\mathrm{pH} 7.4$ (dotted line) and 5.4 (solid line).

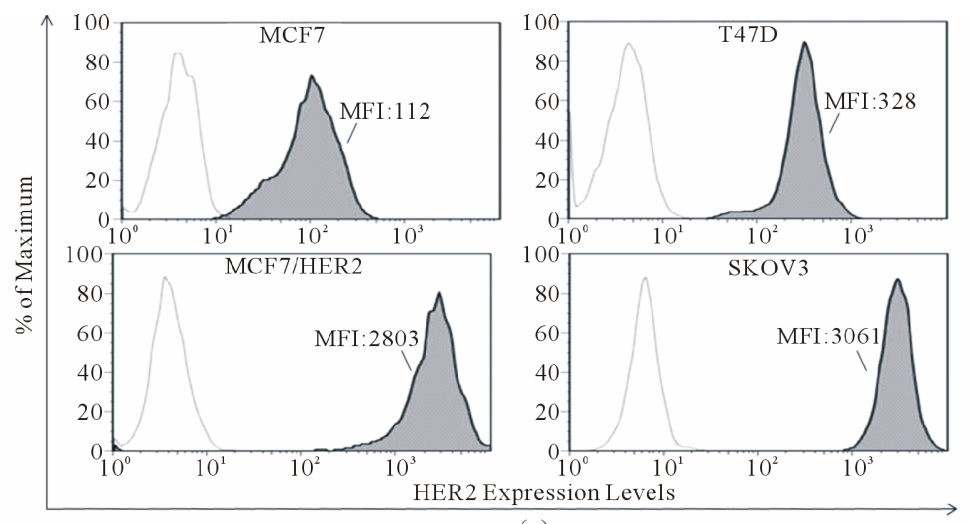

(a)
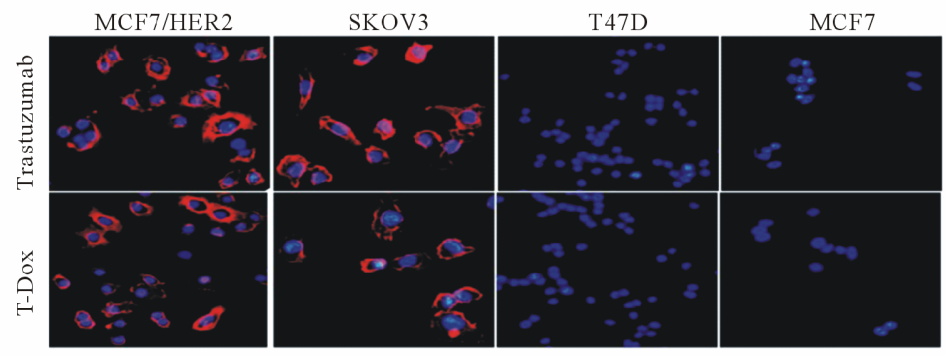

(b)

Figure 2. T-Doxo binding on both cell surface HER2 and recombinant HER2 extracellular protein. (a) HER2 expression in different cancer cells measured by flow cytometry. $\mathrm{X}$-axis indicates fluorescence intensity and $\mathrm{Y}$-axis shows \% of maximum cells in the peak area among the collected cell events. Open peaks indicate cells stained with isotype control antibody and dark area (filled peaks) indicates cells stained with HER2 specific antibody. Cell line and mean fluorescence intensity (MFI) of the dark area are listed in each graph. Higher MFI value indicates higher HER2 expression. (b) T-Dox binding on cell surface HER2 and visualized through anti-human IgG detection antibody conjugated with R-PE. Equivalent concentration of trastuzumab $(5 \mu \mathrm{g} / \mathrm{ml})$ was used as positive control for binding on cell surface HER2. Images shown are the representatives taken under $20 \times$ objective magnification for each cell lines using a fluorescence microscope. 


\subsection{T-Dox Uptake Depends on Both HER2 Expression Levels and HER2 Receptor Endocytosis}

In order to understand how HER2 expression levels affect uptake of T-Dox, we tested the uptake of T-Dox in cancer cells with different levels of HER2. T-Dox uptake by MCF7 and MCF7/HER2 at $37^{\circ} \mathrm{C}$ was detected by the natural red fluorescence of doxorubicin after 5 hours of incubation. Doxorubicin uptake was similar in both low HER2 cells (MCF7) and high HER2 expression MCF7/ HER2 cells (Figure 3(a) upper panel), but uptake of T-Dox was only detectable in MCF7/HER2 cells after 5 hour incubation (Figure 3(a), lower panel). To further compare T-Dox uptake in high HER2 expression cancer cells, we treated Skov3 and MCF7/HER2 cancer cells with different concentrations of Dox or T-Dox and the amount of cell associated T-Dox and Dox was determined by measuring doxorubicin fluorescence signal in the cell lysates. Both T-Dox and doxorubicin showed a concentration-dependent uptake in the two high HER2 expression cancer cells and there was no significant dif- ference of doxorubicin uptake between the two cell lines (Figure 3(b), right panel). MCF7/HER2, however, showed significantly higher levels of cell associated T-Dox than that in Skov3 cells (Figure 3(b), left panel) at the same T-Dox concentrations, even though Skov 3 cells had higher HER2 expression than MCF7/HER2 as shown in Figure 1. The fluorescence signal of doxorubicin inside cells was also visualized by fluorescence microscopy; and MCF7/HER2 cells showed clearly a higher T-Dox uptake than that in Skov3 cells (Supplemental Figure S2). The lower uptake of T-Dox in higher HER2 expressing Skov3 cells indicates that uptake of T-Dox was affected not only by HER2 expression levels but also by other intrinsic HER2 receptor recycling properties.

\subsection{T-Dox Significantly Improved Cancer Cell Proliferation Inhibition in High HER2 Cancer Cells and Exhibited Better Selectivity over Doxorubicin in Low HER2 Cells}

In order to determine cell growth inhibition by T-Dox,
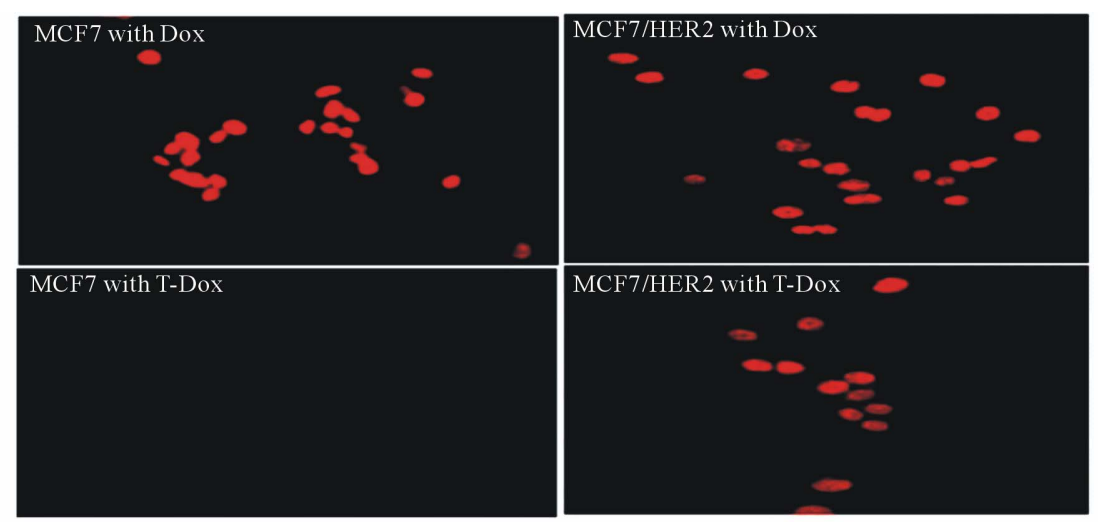

(a)
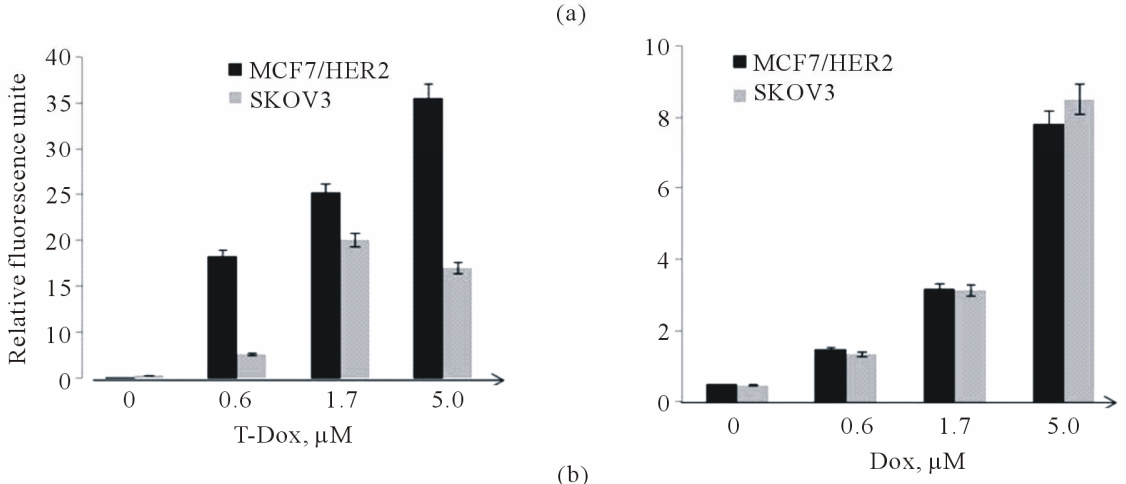

Figure 3. Cell uptake of T-Dox in comparison with doxorubicin in low and high HER2 expression cancer cells. (a) MCF7 (low HER2) and MCF7/HER2 (high HER2) cells were incubated with $5 \mu$ M of doxorubicin or equivalent doxorubicin concentration of T-Dox for $6 \mathrm{~h}$ in a cell culture incubator. The intense red fluorescence of doxorubicin was detected in the nuclei of cells using a fluorescence microscope under $40 \times$ objective magnification. Representative images are shown with cell line and treatment labeled in each image. (b) Uptake of T-Dox and doxorubicin in two high HER2 cancer cell lines after treatment with different concentrations of T-Dox (left graph) and equivalent doxorubicin control (right graph) for 3 hours at $37^{\circ} \mathrm{C}$. Bar graphs show the relative fluorescence units measured in cells (Y-axis) which indicating doxorubicin or T-Dox uptake in cells, $\mathbf{n}=\mathbf{3}$. 
the growth index of different cancer cell lines was monitored continuously using the xCelligence instrument under different concentrations of T-Dox treatment. In comparison with trastuzumab, T-Dox demonstrated signifycantly improved inhibition of cancer cell proliferation in high HER2 (Skov3 and MCF7/HER2) expressing cancer cells (Figure 4, top and middle panels); but trastuzumab alone showed minimal effects on cancer cell proliferation after 2 days of treatment (Figure 4, middle panels). Unexpectedly, T-Dox treatment showed potent inhibition of cell proliferation in the medium to low HER2 expressing T47D cells (Figure 4, bottom panel). In contrast, much lower inhibition of cell proliferation was shown at the same drug concentration in the high HER2 expression Skov3 cells (Figure 4, middle panel). The lower inhibittion by T-Dox in Skov3 cells is consistent with the lower uptake of T-Dox by Skov3 cancer cells as shown in Figure 3(b). These results suggest that HER2 expression level is not the sole factor affecting the potency of T-Dox in cancer cells.

To evaluate the selectivity of T-Dox in different cancer cells, percentage of cell proliferation inhibition was compared between doxorubicin alone and the equivalent doxorubicin concentration of T-Dox conjugate among cancer cells with different HER2 expression levels. Cell proliferation inhibition by T-Dox in low HER2 expressing MCF7 cells was not detectable while doxorubicin showed potent proliferation inhibition of MCF7 cells at the same drug concentration (Figure 5(a)). In contrast, inhibition of cell proliferation by T-Dox in high HER2 expression MCF7/HER2 was similar to that by doxorubicin (Figure 5(a)). Consistent with the results in Figure 4, T-Dox showed higher cell proliferation inhibition in the lower HER2 expressing T47D cells than that in the high HER2 expressing Skov3 cancer cells (Figure 5(b)). Selectivity index (cell inhibition rate of Dox/inhibition
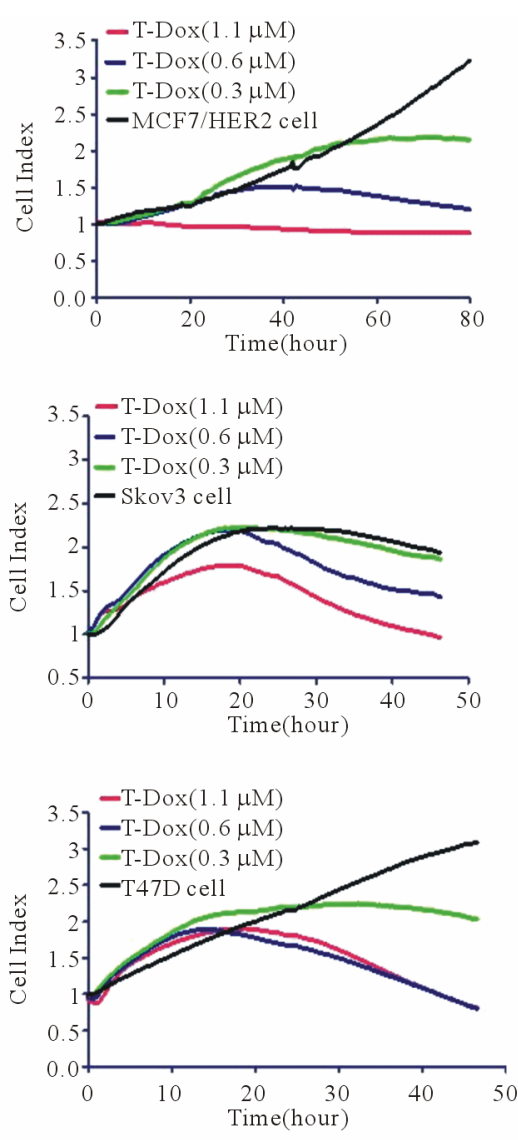

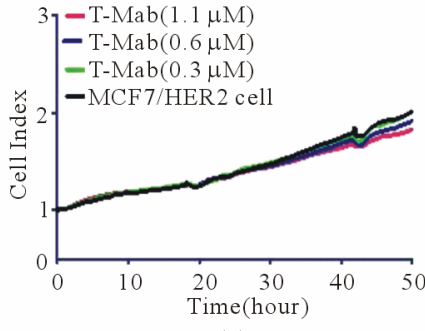

(a)

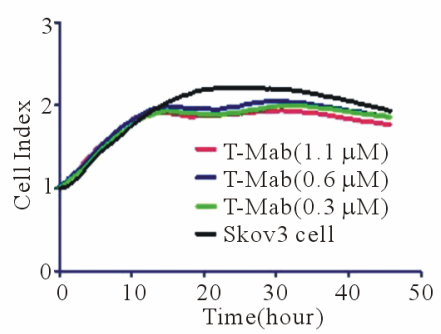

(b)

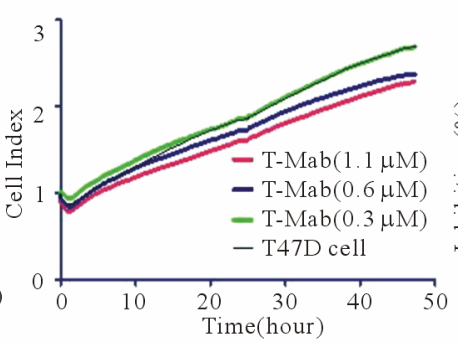

(c)
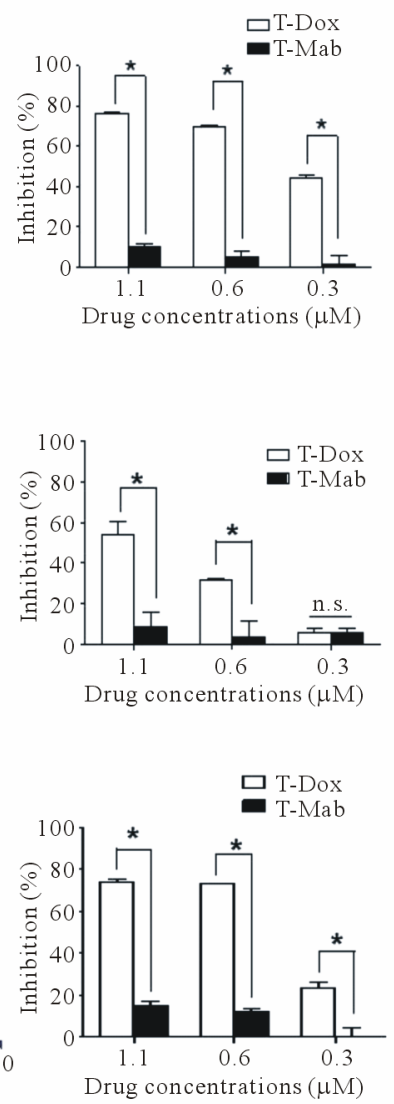

Figure 4. Inhibition of cell proliferation by T-Dox in comparison with trastuzumab in cancer cell lines with different HER2 expression. (a) MCF7/HER2; (b) Skov3; and (c) T47D. The left panel of graphs indicates changes of cell index (Y-axis) over treatment time $(\mathrm{X}$-axis) in cells treated with different concentrations of $\mathrm{T}$-Dox. The middle panel of graphs indicates changes of cell index (Y-axis) over treatment time (X-axis) in cells treated by the equivalent concentrations of trastuzumab (T-Mab). All graphs show means of 3 replications. The right panel of graphs indicates percentage of inhibition (Y-axis) at different concentrations of T-Dox (X-axis) in comparison with equivalent concentrations of trastuzumab (T-Mab). The percentage of inhibition was calculated using the cell index collected at $\mathbf{4 0}$ hours. Cell index of untreated cells was used as no inhibition control. Pairwise Student's t-test was conducted using GraphPad prism 5.0. The n.s. indicates not significant and *indicates $p<0.05$. 


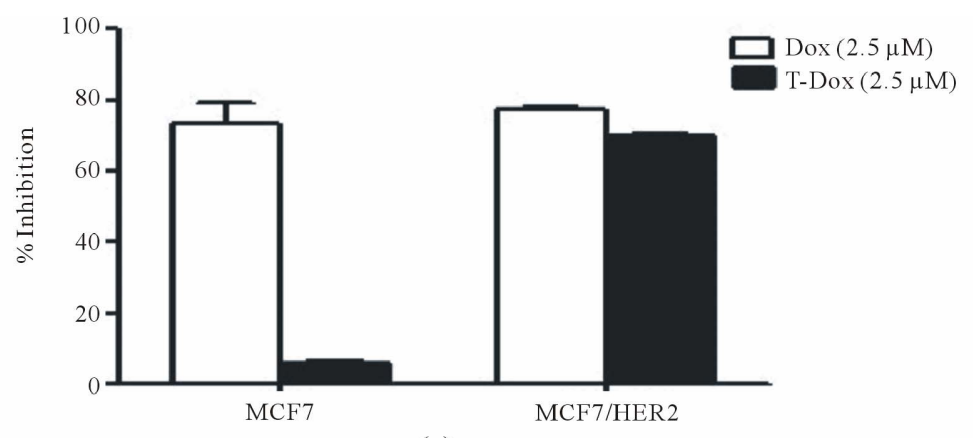

(a)

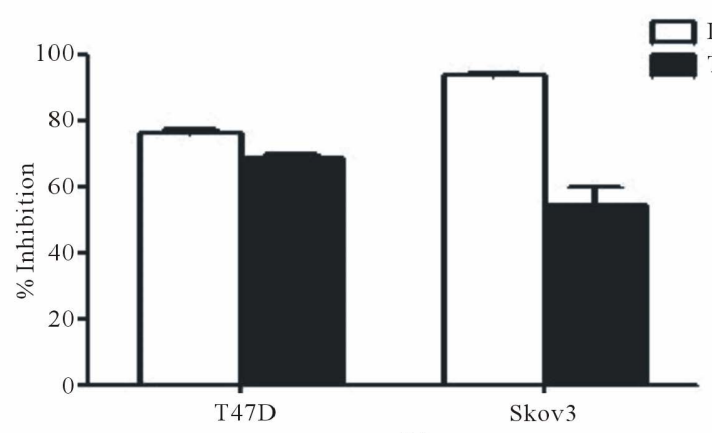

(b)

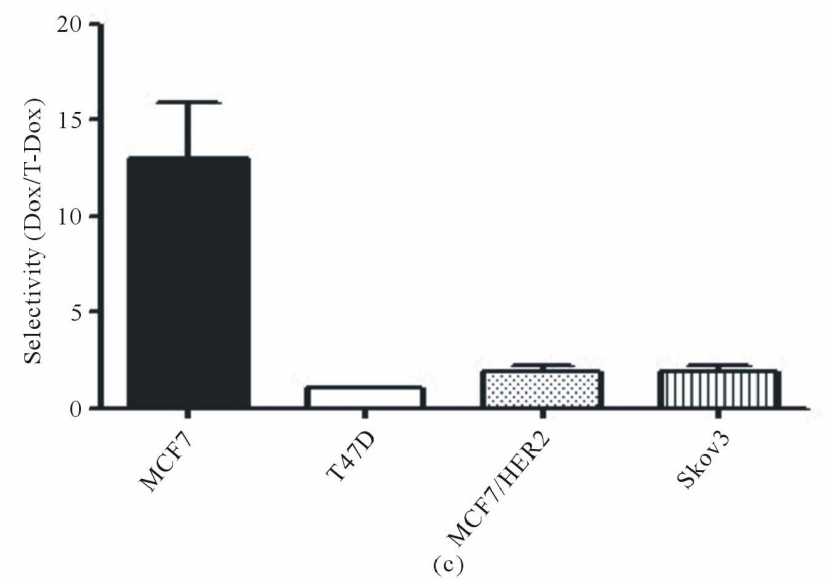

Figure 5. Differential inhibition of cancer cell proliferation by T-Dox in comparison with doxorubicin. (a) Inhibition of cell proliferation by T-Dox in comparison with equivalent concentrations of doxorubicin in low HER2 expression MCF7 and high HER2 expressing MCF7/HER2 cancer cells. Cells were treated with T-Dox at $2.5 \mu \mathrm{M}$ concentration or equivalent doxorubicin for 48 hours. Inhibition of cell growth measured by the cell index was calculated using cell only as control, $n=3$. (b) Inhibition of cell proliferation by T-Dox $(5 \mu M)$ or doxorubicin in medium HER2 expressing T47D cells and high HER2 expression Skov3 cancer cells, $n=3$. (c) Selectivity of T-Dox over doxorubicin (Y-axis) was calculated by (\% of inhibition by doxorubicin $/ \%$ of inhibition by T-Dox) at the same drug concentration. Low HER2 MCF7 cells show highest selectivity while high HER2 expressing cells show selectivity close to 1 indicating similar inhibition of cell proliferation by T-Dox and doxorubicin in high HER2 cancer cells.

rate of T-Dox) in different cancer cell lines is shown in Figure 5(c) and T-Dox showed more than 10 folds better selectivity than doxorubicin alone in low HER2 expression MCF7 cancer cells.

\subsection{T-Dox Significantly Reduced Cytotoxicity of Human Cardiomyocytes}

Human cardiomyocytes had no detectable level of HER2 expression when measured using fluorescence imaging with an anti-HER2 antibody (Figure 6(a)). To determine cytotoxic effects of T-Dox on human cardiomyocytes, viability of primary cardiomycytes was continuously monitored using the $x$ Celligence instrument. There were various degrees of decrease in cell index (reflective of cell viability and growth) in the different treatments over a 70-hour period of the culturing (Figure 6(b)). Com- 
paring with the untreated cardiomyocyte cells, T-Dox and trastuzumab showed minimal effects on cardiomyocyte viability during the first 30-hour exposure at $5 \mu \mathrm{M}$ drug concentration, while doxorubicin showed more than $30 \%$ decrease of cell viability during the same time period at the same drug concentration (Figure 6(c)). After 70 hours of culturing, more than $90 \%$ of cardiomyocytes exhibited cytotoxic effect in the doxorubicin treatment group, but less than $30 \%$ decrease of cell viability was observed in the T-Dox treatment group (Figure 6(c)). Compared to trastuzumab, T-Dox showed a slightly greater effect on cardiomyocyte viability after 70 hours of treatment due to possible presence of free doxorubicin release in the culture.
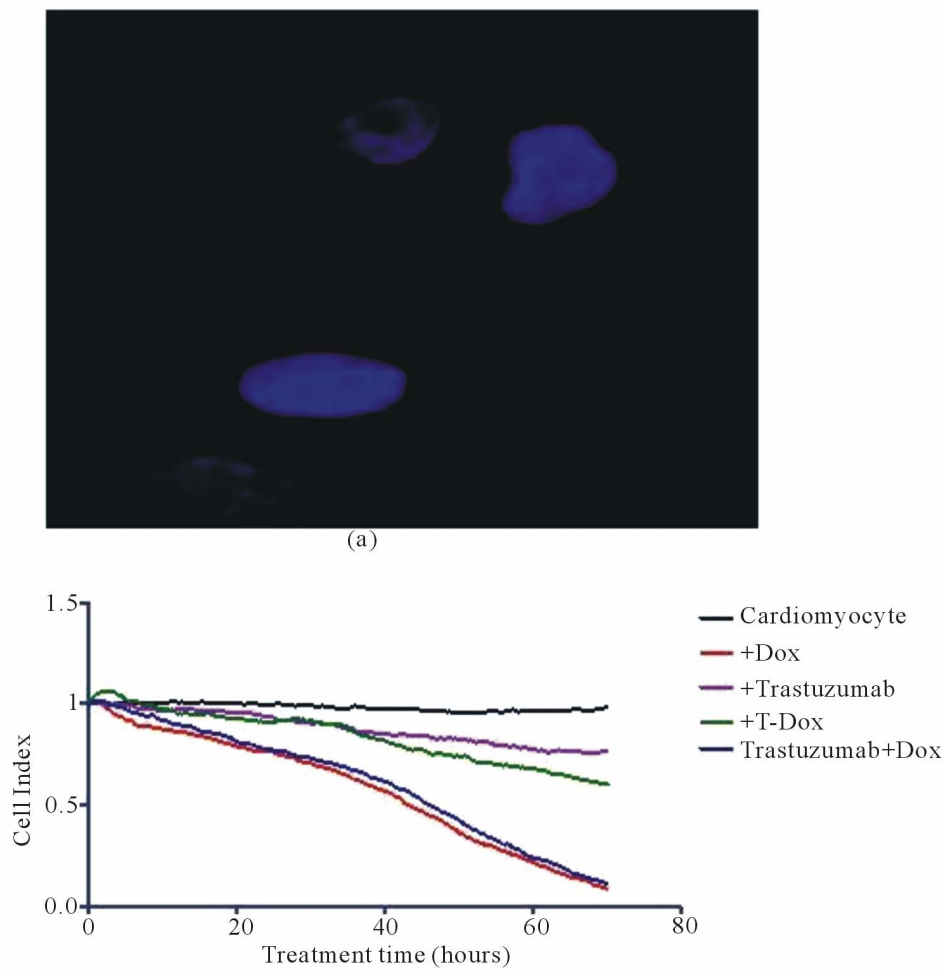

(b)

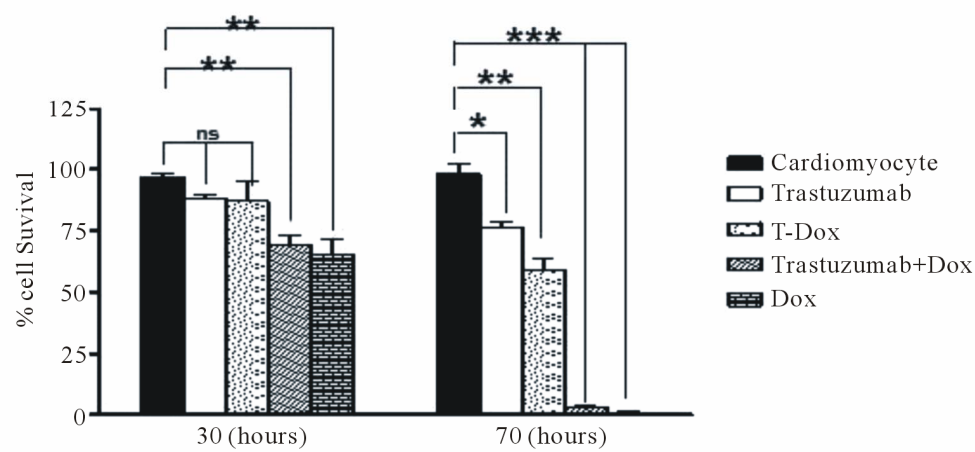

(c)

Figure 6. T-Dox binding and its effects of cell viability of human cardiomyocytes in cell culture treated with $5 \mu$ M of T-Dox in comparison with doxorubicin, trastuzumab or combination of trastuzumab and doxorubicin. (a) Detection of T-Dox binding on human cardiamyocyte cells using the same method described in Figure 2(b) and representative image shows no detectable T-Dox binding on human cardiomyocyte cells and nuclei were stained with DAPI (4',6-Diamidino-2-phenylindole dihydrochloride) dye (blue). (b) Cell index was monitored over a period of 3 days (X-axis, in hours) using xCelligence for monitoring cell viability change ( $\mathrm{Y}$-axis) in different treatment groups. At the time of treatment, cell index was normalized at 1 and the decrease of cell index indicates reducing cell viability. (c) Percentages of viability of human cardiomyocyte cells after $30 \mathrm{~h}$ or $70 \mathrm{~h}$ treatments with T-Dox are shown in $\mathrm{Y}$-axis in comparison with doxorubicin alone, trastuzumab alone, and combination treatment with doxorubicin and trastuzumab (trastuzumab + Dox). Cell index of cardiomyocyte cells was monitored over the entire period of the treatment, $n=3$. 


\section{Discussion}

During preclinical development of the trastuzumab-DM1 (T-DM1) conjugate, it was found that an irreversible thioether linkage between the monoclonal antibody and the maytansinoid thiol derivative DM1, using the maleimidosuccinimidyl linker SMCC, rendered the resultant conjugate clearly superior to disulfide-linked conjugates in terms of in vitro stability, pharmacokinetics and toxicity [29]. Although it was expected that the disulfide linkers would make the corresponding conjugates more prone to reductive cleavage intracellularly, while the thioether linkage would be more resistant to free drug release within tumor cells, the endocytic pathway was shown to be oxidative and the trastuzumab-MCC-DM1 was, in fact, at least as cytotoxic against HER2-expressing cell lines as the disulfide conjugates. Thus, the thioether conjugate was chosen for clinical development of the T-DM1 trastuzumab-drug conjugate. Pursuing an analogous strategy, we chose to couple the carbonyl function of doxorubicin to trastuzumab via a thioether linkage, using the maleimidohydrazide linker $\mathrm{MMCCH}$ (SMCC hydrazide) [22]. Since antibody-dependent cellmediated cytotoxicity (ADCC) has been implicated as a major mechanism of trastuzumab anti-tumor activity in our recent studies and reports by others $[26,28,30]$, we introduced thiols into the immunoglobulin molecules at the sites of free amino functions, using 3-(2-pyridyldithiolpropionic acid)-N-hydroxysuccinimide ester (SPDP), to preserve the integrity of the Fc region and avoid exposing endogenous thiols through reductive dissociation of the IgG monomers. The comparable HER2 binding shown between T-Dox and trastuzumab confirmed the integrity of trastuzumab in the T-Dox conjugate.

Lewis Phillips et al. [29] demonstrated that $>90 \%$ of intact maleimido thioether trastuzumab-DM1 conjugate was recoverable in mouse serum 3 days after intravenous injection of Tmab-MCC-DM1. Our data showed that about half intact maleimido thioether IgG-doxorubicin conjugate remained after in vitro incubation at $37^{\circ} \mathrm{C}$ for 3 days and the difference may be attributable to the nature of the thio-linkage. DM1 is a thiolated derivative of maytansine, an antimitotic compound that inhibits microtubule formation, and SMCC derivatization of the antibody occurs through an epsilon lysyl amino linkage [29]. On the other hand, SMCC hydrazide derivatization of doxorubicin occurs through the drug's carbonyl function, converting the anthracycline 13-keto group into a relatively stable hydrozone [22]. Comparison of our results with those of Lewis Phillips et al. [29] suggests that the amino linkage is more stable than the hydrozone. In addition, differences in assay methods used for determining intact ADC may also contribute to the difference of recovery of intact antibody conjugates. We used desalting column to separate and recover T-Dox in the flow through and there is potential loss of T-Dox in the column which can also contribute to the reduced recovery of intact T-Dox in our results. Nevertheless, since antibody treatment in vivo is generally dosed twice weekly, the stability of T-Dox ( $\mathrm{T}_{1 / 2}$ close to 3 days) should be sufficient for further investigation of the conjugate in an in vivo model.

Mechanisms of action of trastuzumab depend on both engagement of HER2 on cancer cells and engagement of immune effector cell function through its Fc region. Although it is generally considered that trastuzumab functions through engaging the cell-surface HER2 extracellular domain of the receptor, it is poorly understood how internalization of trastuzumab via HER2 contributes to its efficacy even though HER2 downregulation by trastuzumab was proposed as a mechanism of action [31]. Trastuzumab can be internalized through HER2 endocytosis and pulse-chase treatment of high HER2 expressing $\mathrm{SKBr} 3$ cancer cells with trastuzumab showed slow degradation of the antibody (about $40 \%$ turnover rate in 24 hours) in cancer cells [23]. Antibody-drug conjugates depend on antibody-mediated interaction with tumor specific antigen for internalization and delivery of cytotoxic agents into the cells. The trastuzumab drug conjugate T-DM1 has shown positive clinical efficacy for treatment of trastuzumab and laptinib refractory breast cancer [32]. It is assumed that uptake of T-DM1 is mediated by trastuzumab internalization through binding of the HER2 extracellular domain [29]. This study showed that T-Dox uptake had significant differences among high HER2 expression cancer cells and better uptake of T-Dox showed stronger anti-cancer potency.

Internalization of trastuzumab is generally not considered necessary for its mechanism of action. However, uptake of trastuzumab-drug conjugates is an essential step for delivery of cytotoxic drug into HER2 targeted cells. Factors affecting uptake of trastuzumab-drug conjugate can directly impact its anti-cancer efficacy. Our data for the human IgG (as control for trastuzumab) conjugated to doxorubicin (IgG-Dox) in this study showed no detectable binding on HER2 expressing MCF7/HER2 cells and had little effects on cancer cell proliferation (Supplemental Figure S3) even though longer incubation ( $>60$ hours) showed small decrease of cell growth index $(<10 \%$ decrease $)$ possibly due to slow breakage of the conjugate linker of IgG-Dox. The results indicate that the antibody conjugate IgG-Dox is not passively taken up by cancer cells and uptake of T-Dox was mediated specifically through T-Dox binding HER2 on cell surface. In low HER2 expression cells such as MCF7 cancer cells and cardiomyocytes, the T-Dox can provide high selectivity and the data showed multifold ( $>10$ fold) reduction of cytotoxic effects in comparison with doxorubicin 
and combination of doxorubicin with trastuzumab. On the other hand, in high HER2 expressing cancer cells, T-Dox showed superior cytotoxic effects in comparison with trastuzumab. But differences of T-Dox uptake among high HER2 expressing cells (Skov3 vs MCF7/ HER2) indicate that other intrinsic cellular differences, such as HER2 recycling properties, in addition to HER2 expression levels can also affect T-Dox uptake, therefore impact the anti-cancer potency. T-Dox uptake showed positive correlation with anti-cancer potency, suggesting that T-Dox uptake may serve as a potential predictive marker for sensitivity to the trastuzumab-drug conjugates in high HER2 cancer cells. Such predictive biomarker should have significant value if similar correlation holds true for T-DM1 in the clinic.

Trastuzumab conjugated with geldanamycin (GA) using a similar thioether linker was reported [33]. GA is an inhibitor of HSP90 and is known to down-regulate HER2 levels by diverting endosomal trafficking of HER2 from recycling back to membrane surface $[23,34]$. A combination study of trastuzumab and 17-AAG (17-(allylamino)17-demethoxygeldanamycin) showed enhanced HER2 down-regulation [35]. Since uptake of trastuzumab-drug conjugates is dependent on binding of HER2 for internalization, down-regulation of HER2 by GA may decrease uptake of trastuzumab-GA conjugate. Our data showed that short-term treatment with a combination of GA and trastuzumab decreased HER2 level on cell surface (decreased staining intensity) but increased relative accumulation of trastuzumab inside of cell (Supplemental Figure S4). The counter-effect of GA on cell-surface HER2 levels may lead to reduced uptake of trastuzumab conjugate in longer term and complicates its effect on anti-cancer function.

Our study using multiple cancer cell lines with a diverse range of HER2 expression showed that the trastuzumab-doxorubicin conjugate (T-Dox) retained the selectivity of trastuzumab for targeting HER2-expressing cells and reduced the cytotoxic effects of doxorubicin in low HER2 expressing human cardiomyocytes. The selectivity of T-Dox supports the previous reports for trastuzumab ADCs and showed similarity to other trastuzumab conjugates in HER2 targeted delivery and reduced general cytotoxicity in low HER2 cells [20]. Since doxorubicin is an established chemotherapeutic agent, T-Dox should provide unique value for cancer patients with high HER2 expression, who showed benefit from doxorubicin, but also exhibited cardiotoxicity. Compared to trastuzumab alone, T-Dox showed a multifold improvement in anti-cancer potency not only in HER2 overexpressing cancer cells, but also in cells with moderate HER2 expressing T47D cancer cells. Therefore, T-Dox demonstrated benefits of both trastuzumab for cancer targeting specificity and doxorubicin for potent anti-cancer effi- cacy. Further investigation of T-Dox using in vivo models is warranted.

\section{Acknowledgements}

We thank Dr. Y. J. Geng for providing human primary cardiomyocyte cells and Dr. D. Danila for her technical assistance on HPLC separation.

\section{REFERENCES}

[1] L. Gianni, E. Salvatorelli and G. Minotti, "Anthracycline Cardiotoxicity in Breast Cancer Patients: Synergism with Trastuzumab and Taxanes," Cardiovascular Toxicology, Vol. 7, No. 2, 2007, pp. 67-71. doi:10.1007/s12012-007-0013-5

[2] K. Y. Chan, P. Xiang, L. Zhou, K. Li, P. C. Ng, C. C. Wang, L. Zhang, H. Y. Deng, N. H. Pong, H. Zhao, W. Y. Chan and R. Y. Sung, "Thrombopoietin Protects against Doxorubicin-Induced Cardiomyopathy, Improves Cardiac Function, and Reversely Alters Specific Signalling Networks," European Journal of Heart Failure, Vol. 13, No. 4, 2011, pp. 366-376. doi:10.1093/eurjhf/hfr001

[3] C. H. Chen, N. V. Cuong, Y. T. Chen, R. C. So, I. Liau and M. F. Hsieh, "Overcoming Multidrug Resistance of Breast Cancer Cells by the Micellar Doxorubicin Nanoparticles of MPEG-PCL-Graft-Cellulose," Journal of Nanoscience and Nanotechnology, Vol. 11, No. 1, 2011, pp. 53-60. doi:10.1166/jnn.2011.3102

[4] A. Gabizon, H. Shmeeda and Y. Barenholz, "Pharmacokinetics of Pegylated Liposomal Doxorubicin: Review of Animal and Human Studies," Clinical Pharmacokinetics, Vol. 42, No. 5, 2003, pp. 419-436. doi:10.2165/00003088-200342050-00002

[5] H. D. King, A. J. Staab, K. Pham-Kaplita, D. Yurgaitis, R. A. Firestone, S. J. Lasch and P. A. Trail, "BR96 Conjugates of Highly Potent Anthracyclines," Bioorganic \& Medicinal Chemistry Letters, Vol. 13, No. 13, 2003, pp. 2119-2122. doi:10.1016/S0960-894X(03)00375-5

[6] S. Meschini, M. Condello, P. Lista, B. Vincenzi, A. Baldi, G. Citro, G. Arancia and E. P. Spugnini, "Electroporation Adopting Trains of Biphasic Pulses Enhances in Vitro and in Vivo the Cytotoxic Effect of Doxorubicin on Multidrug Resistant Colon Adenocarcinoma Cells (LoVo)," European Journal of Cancer, Vol. 48, No. 14, 2012, pp. 2236-2243. doi:10.1016/j.ejca.2011.11.031

[7] M. E. O'Brien, N. Wigler, M. Inbar, R. Rosso, E. Grischke, A. Santoro, R. Catane, D. G. Kieback, P. Tomczak, S. P. Ackland, F. Orlandi, L. Mellars, L. Alland and C. Tendler, "Reduced Cardiotoxicity and Comparable Efficacy in a Phase III Trial of Pegylated Liposomal Doxorubicin $\mathrm{HCl}$ (CAELYX/Doxil) versus Conventional Doxorubicin for First-Line Treatment of Metastatic Breast Cancer," Annals of Oncology, Vol. 15, No. 3, 2004, pp. 440-449. doi:10.1093/annonc/mdh097

[8] J. A. O'Shaughnessy, "Pegylated Liposomal Doxorubicin in the Treatment of Breast Cancer," Clinical Breast Cancer, Vol. 4, No. 5, 2003, pp. 318-328.

doi:10.3816/CBC.2003.n.037 
[9] P. A. Trail, H. D. King and G. M. Dubowchik, "Monoclonal Antibody Drug Immunoconjugates for Targeted Treatment of Cancer," Cancer Immunol Immunother, Vol. 52, No. 5, 2003, pp. 328-337.

[10] Y. Y. Diao, H. Y. Li, Y. H. Fu, M. Han, Y. L. Hu, H. L. Jiang, Y. Tsutsumi, Q. C. Wei, D. W. Chen and J. Q. Gao, "Doxorubicin-Loaded PEG-PCL Copolymer Micelles Enhance Cytotoxicity and Intracellular Accumulation of Doxorubicin in Adriamycin-Resistant Tumor Cells," International Journal of Nanomedicine, Vol. 6, No. 9, 2011, pp. 1955-1962.

[11] B. Guo, H. L. Zhu, S. X. Li, X. C. Lu and H. Fan, "Individualized Liposomal Doxorubicin-Based Treatment in Elderly Patients with Non-Hodgkin's Lymphoma," Onkologie, Vol. 34, No. 4, 2011, pp. 184-188. doi:10.1159/000327008

[12] I. Kim, H. J. Byeon, T. H. Kim, E. S. Lee, K. T. Oh, B. S. Shin, K. C. Lee and Y. S. Youn, "Doxorubicin-Loaded Highly Porous Large PLGA Microparticles as a Sustained-Release Inhalation System for the Treatment of Metastatic Lung Cancer," Biomaterials, Vol. 33, No. 2, 2012, pp. 5574-5583. doi:10.1016/j.biomaterials.2012.04.018

[13] X. W. Ma, Y. L. Zhao and X. J. Liang, "Nanodiamond Delivery Circumvents Tumor Resistance to Doxorubicin," Acta Pharmacologica Sinica, Vol. 32, No. 5, 2011, pp. 543-544. doi:10.1038/aps.2011.58

[14] J. Niu, Z. Su, Y. Xiao, A. Huang, H. Li, X. Bao, S. Li, Y. Chen, M. Sun and Q. Ping, "Octreotide-Modified and PH-Triggering Polymeric Micelles Loaded with Doxorubicin for Tumor Targeting Delivery," European Journal of Pharmaceutical Sciences, Vol. 45, No. 1-2, 2011, pp. 216-226. doi:10.1016/j.ejps.2011.11.013

[15] L. Zhao, L. Zhu, F. Liu, C. Liu, D. Shan, Q. Wang, C. Zhang, J. Li, J. Liu, X. Qu and Z. Yang, "pH Triggered Injectable Amphiphilic Hydrogel Containing Doxorubicin and Paclitaxel," International Journal of Pharmaceutics, Vol. 410, No. 1-2, 2011, pp. 83-91. doi:10.1016/j.ijpharm.2011.03.034

[16] J. G. Reynolds, E. Geretti, B. S. Hendriks, H. Lee, S. C. Leonard, S. G. Klinz, C. O. Noble, P. B. Lucker, P. W. Zandstra, D. C. Drummond, K. J. Olivier Jr., U. B. Nielsen, C. Niyikiza, S. V. Agresta and T. J. Wickham, "HER2Targeted Liposomal Doxorubicin Displays Enhanced Anti-Tumorigenic Effects without Associated Cardiotoxicity," Toxicology and Applied Pharmacology, Vol. 262, No. 1, 2012, pp. 1-10. doi:10.1016/j.taap.2012.04.008

[17] A. G. Garcia, H. Nedev, K. Bijian, J. Su, M. A. AlaouiJamali and H. U. Saragovi, "Reduced in Vivo Lung Metastasis of a Breast Cancer Cell Line after Treatment with Herceptin MAB Conjugated to Chemotherapeutic Drugs," Oncogene, Vol. 283, No. 7, 2012, pp. 1-7.

[18] E. L. Sievers and P. D. Senter, "Antibody-Drug Conjugates in Cancer Therapy," Annual Review of Medicine, Vol. 64, No. 10, 2012, pp. 15-29.

[19] M. Y. Yeh, S. R. Roffler and M. H. Yu, "Doxorubicin: Monoclonal Antibody Conjugate for Therapy of Human Cervical Carcinoma," International Journal of Cancer, Vol. 51, No. 2, 1992, pp. 274-282. doi:10.1002/ijc. 2910510217

[20] H. A. Burris 3rd, J. Tibbitts, S. N. Holden, M. X. Sliwkowski and G. D. Lewis Phillips, "Trastuzumab Emtansine (T-DM1): A Novel Agent for Targeting HER2+ Breast Cancer," Clinical Breast Cancer, Vol. 11, No. 5, 2011, pp. 275-282. doi:10.1016/j.clbc.2011.03.018

[21] D. J. Slamon, B. Leyland-Jones, S. Shak, H. Fuchs, V. Paton, A. Bajamonde, T. Fleming, W. Eiermann, J. Wolter, M. Pegram, J. Baselga and L. Norton, "Use of Chemotherapy plus a Monoclonal Antibody against HER2 for Metastatic Breast Cancer That Overexpresses HER2," The New England Journal of Medicine, Vol. 344, No. 11, 2001, pp. 783-792. doi:10.1056/NEJM200103153441101

[22] G. L. Griffiths, M. J. Mattes, R. Stein, S. V. Govindan, I. D. Horak, H. J. Hansen and D. M. Goldenberg, "Cure of SCID Mice Bearing Human B-Lymphoma Xenografts by an Anti-CD74 Antibody-Anthracycline Drug Conjugate," Clinical Cancer Research, Vol. 9, No. 17, 2003, pp. 6567-6571.

[23] C. D. Austin, A. M. De Maziere, P. I. Pisacane, S. M. van Dijk, C. Eigenbrot, M. X. Sliwkowski, J. Klumperman and R. H. Scheller, "Endocytosis and Sorting of ErbB2 and the Site of Action of Cancer Therapeutics Trastuzumab and Geldanamycin," Molecular Biology of the Cell, Vol. 15, No. 12, 2004, pp. 5268-5282. doi:10.1091/mbc.E04-07-0591

[24] H. K. Erickson, P. U. Park, W. C. Widdison, Y. V. Kovtun, L. M. Garrett, K. Hoffman, R. J. Lutz, V. S. Goldmacher and W. A. Blattler, "Antibody-Maytansinoid Conjugates Are Activated in Targeted Cancer Cells by Lysosomal Degradation and Linker-Dependent Intracellular Processing," Cancer Research, Vol. 66, No. 8, 2006, pp. 4426-4433. doi:10.1158/0008-5472.CAN-05-4489

[25] S. Ha, Y. Ou, J. Vlasak, Y. Li, S. Wang, K. Vo, Y. Du, A. Mach, Y. Fang and N. Zhang, "Isolation and Characterization of IgG1 with Asymmetrical Fc Glycosylation," Glycobiology, Vol. 21, No. 8, 2011, pp. 1087-1096. doi:10.1093/glycob/cwr047

[26] X. Fan, R. J. Brezski, M. Fa, H. Deng, A. Oberholtzer, A. Gonzalez, W. P. Dubinsky, W. R. Strohl, R. E. Jordan, N. Zhang and Z. An, "A Single Proteolytic Cleavage within the Lower Hinge of Trastuzumab Reduces Immune Effector Function and in Vivo Efficacy," Breast Cancer Research, Vol. 14, No. 4, 2012, p. R116. doi: $10.1186 / \mathrm{bcr} 3240$

[27] M. E. Klegerman, A. J. Hamilton, S. L. Huang, S. D. Tiukinhoy, A. A. Khan, R. C. MacDonald and D. D. McPherson, "Quantitative Immunoblot Assay for Assessment of Liposomal Antibody Conjugation Efficiency," Analytical Biochemistry, Vol. 300, No. 1, 2002, pp. 46-52. doi:10.1006/abio.2001.5443

[28] N. Zhang, L. Liu, C. D. Dumitru, N. R. Cummings, M. Cukan, Y. Jiang, Y. Li, F. Li, T. Mitchell, M. R. Mallem, Y. Ou, R. N. Patel, K. Vo, H. Wang, I. Burnina, B. K. Choi, H. E. Huber, T. A. Stadheim and D. Zha, "Glycoengineered Pichia Produced Anti-HER2 is Comparable to Trastuzumab in Preclinical Study," Mabs, Vol. 3, No. 3, 2011, pp. 289-298.

[29] G. D. Lewis Phillips, G. Li, D. L. Dugger, L. M. Crocker, 
K. L. Parsons, E. Mai, W. A. Blattler, J. M. Lambert, R. V. Chari, R. J. Lutz, W. L. Wong, F. S. Jacobson, H. Koeppen, R. H. Schwall, S. R. Kenkare-Mitra, S. D. Spencer and M. X. Sliwkowski, "Targeting HER2-Positive Breast Cancer with Trastuzumab-DM1, an AntibodyCytotoxic Drug Conjugate," Cancer Research, Vol. 68, No. 22, 2008, pp. 9280-9290.

doi:10.1158/0008-5472.CAN-08-1776

[30] N. L. Spector and K. L. Blackwell, "Understanding the Mechanisms behind Trastuzumab Therapy for Human Epidermal Growth Factor Receptor 2-Positive Breast Cancer," Journal of Clinical Oncology, Vol. 27, No. 34, 2009, pp. 5838-5847. doi:10.1200/JCO.2009.22.1507

[31] M. X. Sliwkowski, J. A. Lofgren, G. D. Lewis, T. E. Hotaling, B. M. Fendly and J. A. Fox, "Nonclinical Studies Addressing the Mechanism of Action of Trastuzumab (Herceptin)," Seminars in Oncology, Vol. 26, No. 4, 1999, pp. 60-70.

[32] P. M. LoRusso, D. Weiss, E. Guardino, S. Girish and M. X. Sliwkowski, "Trastuzumab Emtansine: A Unique Antibody-Drug Conjugate in Development for Human Epidermal Growth Factor Receptor 2-Positive Cancer," Cli- nical Cancer Research, Vol. 17, No. 20, 2011, pp. 6437 6447. doi:10.1158/1078-0432.CCR-11-0762

[33] R. Mandler, H. Kobayashi, E.R. Hinson, M. W. Brechbiel and T. A. Waldmann, "Herceptin-Geldanamycin Immunoconjugates: Pharmacokinetics, Biodistribution, and Enhanced Antitumor Activity," Cancer Research, Vol. 64, No. 9, 2004, pp. 1460-1467. doi:10.1158/0008-5472.CAN-03-2485

[34] C. Marx, J. M. Held, B. W. Gibson and C. C. Benz, "ErbB2 Trafficking and Degradation Associated with K48 and K63 Polyubiquitination," Cancer Research, Vol. 70, No. 22, 2010, pp. 3709-3717. doi:10.1158/0008-5472.CAN-09-3768

[35] S. M. Raja, R. J. Clubb, M. Bhattacharyya, M. Dimri, H. Cheng, W. Pan, C. Ortega-Cava, A. Lakku-Reddi, M. Naramura, V. Band and H. Band, "A Combination of Trastuzumab and 17-AAG Induces Enhanced Ubiquitinylation and Lysosomal Pathway-Dependent ErbB2 Degradation and Cytotoxicity in ErbB2-Overexpressing Breast Cancer Cells," Cancer Biology \& Therapy, Vol. 7, No. 10, 2008, pp. 1630-1640. doi:10.4161/cbt.7.10.6585 


\section{Supplements}

Table S1. IgG-Doxorubicin conjugation characteristics.

\begin{tabular}{ccccccc}
\hline & & \multicolumn{2}{c}{$[\mathrm{lgG}]$} & \multicolumn{2}{c}{ [Doxorubicin] } & Dox.lgG Molar Ratio \\
\cline { 3 - 5 } Preparation Batches & immunoglobulin & $\mu \mathrm{g} / \mathrm{ml}$ & $\mu \mathrm{M}$ & $\mu \mathrm{g} / \mathrm{ml}$ & $\mu \mathrm{M}$ & 4.3 \\
\cline { 3 - 5 } IgG-Dox, batch1 & Human $\operatorname{lgG}$ & 308 & 2.1 & 5.1 & 8.8 & 4.7 \\
IgG-Dox, batch2 & Human $\operatorname{lgG}$ & 373 & 2.5 & 6.8 & 11.8 & 4.6 \\
T-Dox, batch1 & Trastuzumab & 132 & 0.9 & 2.4 & 4.1 & 4.7 \\
T-Dox, batch2 & Trastuzumab & 518 & 3.5 & 9.4 & 16.2 & 4.7 \\
\hline
\end{tabular}
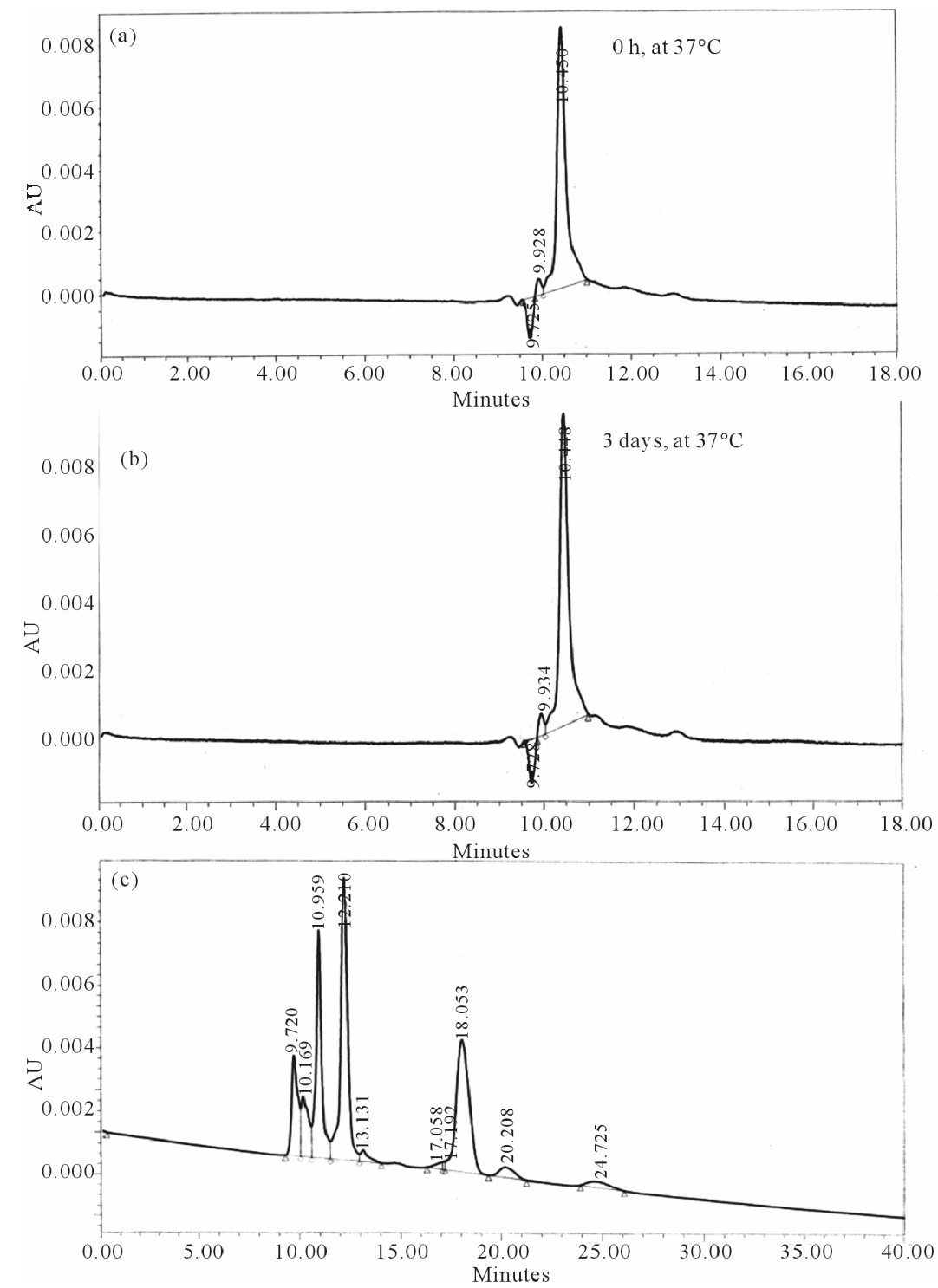

Figure S1. Stability of T-Dox at $37^{\circ}$ and pH 7.4 as determined by size exclusion HPLC. (a). T-Dox preparation was analyzed at 0 hour at $37^{\circ} \mathrm{C}$ after dissolved in solution and intact T-Dox was eluted at 10.5 minutes. (b). Aliquot of T-Dox was incubated for 3 days at $37^{\circ} \mathrm{C}$ after dissolved in PBS and one elution peak at 10.5 minutes was detected indicating T-Dox retained intact IgG. (c). Mixture of thyroglobulin (670 kDa), bovine gamma globulin (150 kDa), ovalbumin (45 kDa), equine myoglobin (16.9 kDa) and vitamin $B_{12}(1.35 \mathrm{kDa})$ was separated in the column conditions for calibration. There was a linear log-log relationship between molecular weight and elution time from 9.72 to 20.2 minutes and the second major elution peak at 10.9 minutes corresponding to $150 \mathrm{kDa}$ molecular weight of the IgG antibody. 


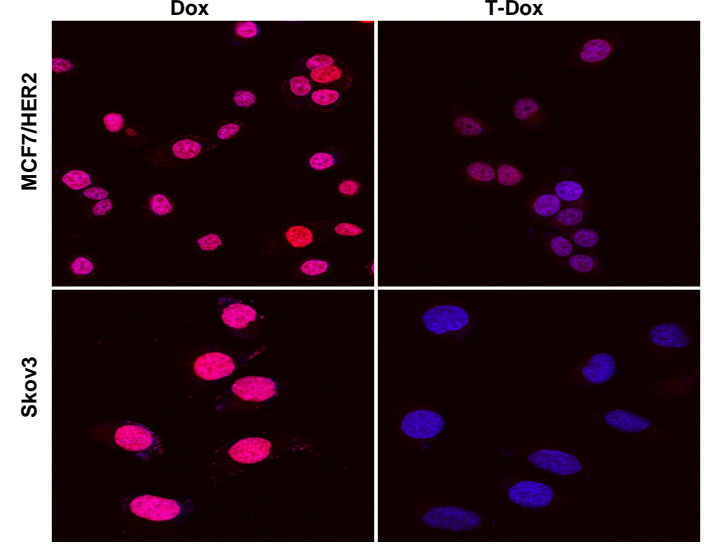

Figure S2. Detection of T-Dox and Dox nucleus uptake in two high HER2 expression cancer cell lines, MCF7/HER2 and Skov3. DAPI stained nuclei are in blue color and pink color shows the merge of blue nuclei with the natural red fluorescence from doxorubicin. Cancer cells were incubated with T-Dox or equivalent doxorubicin at $1 \mu \mathrm{M}$ for 3 hours and imaged using a confocal fluorescence microscope under $63 \times$ objective magnification. Representative images are shown for MCF7/HER2 in upper panel and Skov3 in lower panel.

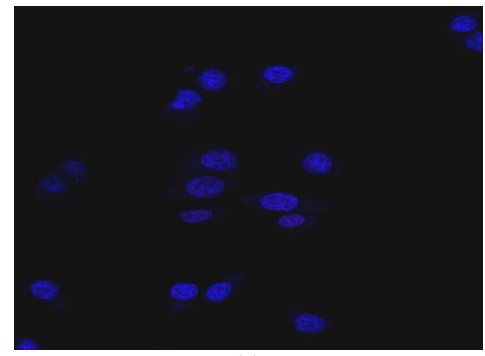

(a)

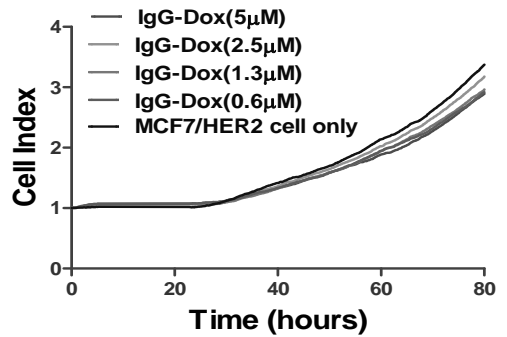

(b)

Figure S3. IgG-Dox binding and cytotoxic effects in high HER2 expression MCF7/HER2 cancer cells. (a) There was no detectable IgG-Dox binding on MCF7/HER2 cells after incubation with $1 \mu \mathrm{g} / \mathrm{ml}$ IgG-Dox and detected with antihuman Fc conjugated with phycoerythrin (PE) (red color). The stained cells were imaged under $40 \times$ objective magnification and representative image is shown. DAPI stained nuclei are in blue color; (b) The high HER2 MCF7/HER2 cancer cells were cultured in the presence of different concentrations of doxorubicin equivalent IgG-Dox (0 - $5 \mu \mathrm{M})$ and cell index was monitored continuously using xCELLigence instrument. There were no significant cell index differences in the presence and absence IgG-Dox indicating that there was no passive uptake of IgG-Dox.

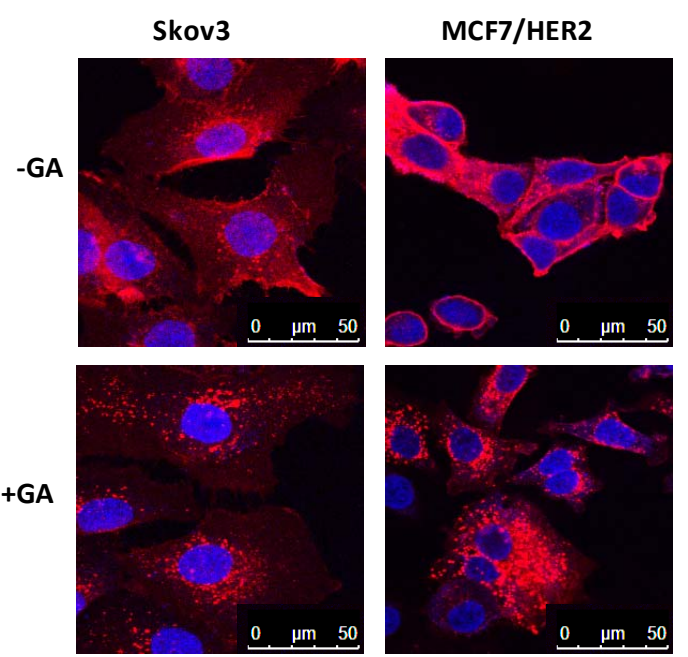

Figure S4. Effects of geldanamycin (GA) treatment on trastuzumab conjugate binding on cell surface HER2 for internalization. Trastuzumab was conjugated with R-PE according to manufacturer's instruction (Invitrogen) and R-PE conjugated trastuzumab $(1 \mu \mathrm{g} / \mathrm{ml})$ was incubated in the absence (upper panel) and presence (lower panel) of 1 $\mu M$ GA for 3 hours at $37^{\circ} \mathrm{C}$ in a cell culture incubator. Cells were fixed and imaged using a confocal fluorescence microscope as described in the Method section. The red fluorescence staining detects both cell surface binding and internalization of trastuzumab-R-PE. Cell surface binding on HER2 by trastuzumab-R-PE is indicated by the red fluorescence staining at the edge of cells and the red fluorescence inside of cells (surrounding the blue nuclei stain) indicates HER2 mediated internalization of trastuzumabR-PE. In the presence of GA, representative cell images show lower red fluorescence intensity on cell surface (edge of cells) in the high HER2 expression cell lines Skov3 and MCF7/HER2. In the absence of GA treatment, higher red fluorescence intensity inside of cells (surrounding nuclei) was observed in both high HER2 expression cell lines, Skov3 and MCF7/HER2. 